VERA LÚCIA FIGUEREDO BORGES ${ }^{1}$

Sebastião Frettas de Mederros ${ }^{2}$

Artigo original

Palavras-chave

Sexualidade/fisiologia

Saúde da mulher

Climatério

Pós-menopausa

Comportamento sexual

Questionário

Estudos de validação

Keywords

Sexuality/physiology

Women's health

Climacteric

Postmenopause

Sexual behavior

Questionnaire

Validation studies

\section{Validação de questionário para avaliar a função sexual feminina após menopausa}

\author{
Validation of a questionnaire to evaluate the female sexual function \\ in postmenopausal women
}

\section{Resumo}

OBJETIVO: elaborar e validar um questionário para medir os diferentes domínios da função sexual nas mulheres brasileiras pós-menopausa. MÉTODOS: participaram deste estudo 251 mulheres entre 2 e 15 anos após menopausa. Foi examinada a reprodutibilidade/confiabilidade do instrumento pelos coeficientes de correlação linear, intraclasse e concordância de Lin. A consistência interna foi examinada pelo coeficiente $\alpha$ de Cronbach. A verificação da face, conteúdo e construto seguiram diretrizes teóricas clássicas para validação de testes. RESULTADOS: foi gerado um instrumento inicial com 57 itens estruturados em nove domínios. Foram eliminados 14 (24,5\%) questões por baixa correlação com a escala total ou pequeno poder de discriminação. A forma final com 43 itens demonstrou excelente reprodutibilidade ( $\mathrm{r}=0,719 ; \mathrm{IC} 95 \%=0,690-0,750 ; \mathrm{pc}=0,887 ; \mathrm{IC} 95 \%=0,850-0,930 ; \mathrm{p}<0,001)$ e confiabilidade. A consistência interna da escala foi excelente ( $\alpha$ de Cronbach 0,951) e cerca de $60 \%$ dos avaliadores confirmaram as validades de face e conteúdo. $\bigcirc$ exame da validade de construto mostrou adequação do instrumento $(\alpha=0,951)$. CONCLUSÕES: concluiu-se que o questionário proposto é adequado para examinar a função sexual feminina em mulheres pós-menopausa.

\section{Abstract}

PURPOSE: to generate and validate a proper questionnaire to evaluate the sexual function in post-menopause women. METHODS: 251 women, within 2 to 15 years postmenopause, were included in the study. Questionnaire's reproductibility/ reliability was evaluated by Pearson, intraclass and Lin's correlation coefficients. The internal consistance was examined by the Cronbach's alpha coefficient. Classical item theory guidelines were used for face, content and construct validation. RESULTS: an instrument with 57 items and nine domains was generated. Fourteen questions (24.5\%) were eliminated by either poor correlation with the scale or low discriminative power. The final version with 43 items has shown good reproductibility $(r=0.719,95 \% \mathrm{Cl}=0.690-0.750 ; \mathrm{pc}=0.887 ; 95 \% \mathrm{Cl}=0.850-0.930 ; \mathrm{p}<0.001)$. Internal consistance was also adequate $(\alpha=0.951)$. About $60 \%$ of the reviewers have confirmed face and content validation. The construct validation was assessed by the Cronbach alpha 0.951. CONCLUSIONS: it was concluded that the new instrument is appropriate for evaluating the sexual function in post-menopause women. (EP 78043-306 - Cuiabá (MT), Brasil Fone: (65) 3322-7342 - Fax: (65) 3623-0079 E-mail: de.medeiros@terra.com.br
Ambulatório de Climatério do Departamento de Ginecologia e Obstetrícia do Hospital Universitário Júlio Müller - Cuiabá (MT), Brasil. 'Médica do Hospital e Maternidade Santa Lúcia - Jaciara (MT), Brasil.

2 Professor Adjunto IV do Hospital Universitário Júlio Müller; Departamento de Ginecologia e Obstetrícia da Faculdade de Ciências Médicas da Universidade Federal de Mato Grosso - UFMT; Diretor do Instituto Tropical de Medicina Reprodutiva e Menopausa Cuiabá (MT), Brasil. 


\section{Introdução}

Mulheres saudáveis, com parceiros adequados, permanecem sexualmente interessadas até o final da vida ${ }^{1,2}$. As mudanças sexuais que podem ocorrer no climatério têm início lento e progressivo, como consequência da diminuição dos hormônios femininos ${ }^{3}$. A resposta sexual pode sofrer algumas alterações, mas não desaparece neste período. A condição pós-menopausa pode inclusive ser vantajosa em algumas culturas, nas quais não há o temor de gravidez e a mulher não dispende tanto tempo para cuidar dos filhos, libertando-se das inibições que antes atrapalhavam sua vida sexual ${ }^{4}$. Variáveis como idade, tempo de relacionamento, aumento das morbidades com diminuição do bem-estar e o próprio hipoestrogenismo da pós-menopausa contribuem para uma possível redução da atividade sexual nesta fase da vida ${ }^{3}$. Estudos etnogeográficos realizados há várias décadas revelaram que em algumas sociedades a posição social e as condições sexuais da mulher melhoram durante seus anos pós-reprodutivos, uma vez que ela se vê liberada das restrições sociais concebidas para controlar a sexualidade feminina ${ }^{1}$. O impacto da cultura, condições físico-psíquicas e fatores associados ao relacionamento são variáveis relevantes que podem afetar a função sexual de um grande número de mulheres após a menopausa, principalmente se a longevidade feminina atual for considerada ${ }^{5,6}$.

A resposta sexual feminina tem pouca espontaneidade, sendo sua dimensão essencialmente dependente de estímulo ${ }^{7}$. Este modelo atualmente proposto, não sequencial, incluindo intimidade emocional e satisfação física como metas, consiste de vários fatores conectados e envolve desejo sexual (espontâneo ou estimulado), excitação, satisfação física, satisfação emocional e intimidade emocional ${ }^{8}$. Qualquer rompimento neste ciclo, incorporando componentes físicos e mentais, resulta em disfunção sexual. Nos Estados Unidos admite-se prevalência de disfunção sexual feminina de $43 \%$ em mulheres entre 18 e 59 anos $^{9}$ Um estudo australiano mostra aumento da disfunção sexual de 42 para $88 \%$ em seguimento de oito anos, em mulheres entre 45 a 55 anos $^{10}$. No Brasil, aproximadamente um terço das mulheres têm desejo sexual hipoativo, $29 \%$ não atingem o orgasmo e $18 \%$ têm dispareunia ${ }^{11}$. Considerando só as mulheres após a menopausa, a proporção não é conhecida.

Não existem informações na literatura nacional examinando especificamente a função sexual das mulheres após a menopausa com instrumentos apropriados. Logo, não se sabe se a função sexual das mulheres climatéricas brasileiras é semelhante à de outras comunidades ${ }^{12}$. Estudos com resultados confiáveis neste contexto dependem do emprego de escalas precisas e previamente validadas para medir a função sexual feminina. No presente estudo, optou-se por validar questionário novo e específico, em vez de traduzir e validar um instrumento já validado. Isto porque embora existam instrumentos para medir a existência e severidade da maioria dos sintomas associados à menopausa, não existe escala específica validada para medir a satisfação sexual da mulher após a introdução da classificação de Basson ${ }^{7,13}$ neste período de vida. Alguns centros têm tentado fazer esta avaliação usando questionários não submetidos aos rigores dos processos de validação, pilar para a validade interna de qualquer relatório científico. Além disso, os instrumentos disponíveis validados com outros modelos de resposta sexual, em outras populações e outros idiomas, podem não ser adequados para uso em todos os países ${ }^{14}$. Alguns questionários, validados de outras línguas, já são disponíveis em português para avaliar a função sexual feminina, no entanto incluem mulheres em todas as faixas etárias e antecedem a classificação de Basson ${ }^{15,16}$.

\section{Métodos}

O presente estudo seguiu diretrizes e metodologia teórica clássica dos testes utilizada para validação de questionários: verificação da confiabilidade, reprodutibilidade, homogeneidade, consistência interna e validades de face, conteúdo e construto de cada item em determinado domínio e do instrumento como um todo ${ }^{17-20}$. Descreve ainda, em sequência, os passos envolvidos no desenvolvimento e teste do instrumento.

Um total de 251 mulheres com idade média de 54 anos, 2 a 15 anos após menopausa natural, não randomizadas, atendidas no Ambulatório de Climatério do Departamento de Ginecologia e Obstetrícia do Hospital Universitário Júlio Müller ou encaminhadas a este serviço pela técnica "bola-de-neve" participam deste estudo descritivo de corte transversal. Pacientes sem atividade sexual, hipertensas, diabéticas, cardiopatas, usuárias de agonistas da dopamina, andrógenos, corticosteroides e portadoras de doenças músculoesqueléticas com limitação dos movimentos foram consideradas inelegíveis. Foram incluídas mulheres com atividade sexual estável e regular, independente do estado civil ou opção sexual. Todas que aceitaram participar do estudo assinaram o termo de consentimento livre e esclarecido aprovado pelo Comitê de Ética e Pesquisa do Hospital Universitário Júlio Müller.

Dentre as 251 participantes, 55 delas, com idade média de 53,2 $\pm 5,6$ anos, participaram do teste-reteste da primeira versão. Destas 55 mulheres na pós-menopausa, $70,9 \%$ eram casadas e $23,6 \%$ eram separadas ou separadas em segunda união estável; 54,5\% eram brancas, 23,6\% negras, $10,9 \%$ amarelas, $3,6 \%$ indígenas e 7,2\% miscigenadas. Considerando a escolaridade, $5,4 \%$ eram analfabetas, 43,6\% não tinham concluído o Ensino Fundamental, 12,7\% tinham concluído o Ensino Fundamental, 9,1\% 
não concluíram o Ensino Médio e 12,7\% tinham o Ensino Médio concluído. Apenas 16,3\% tinham concluído o Ensino Superior. Mais da metade $(57,1 \%)$ exercia atividades domésticas, 9,1\% dedicavam-se ao comércio, 7,7\% eram professoras, $3,6 \%$ faziam serviços gerais, $3,1 \%$ eram lavradoras e 28,5\% tinham outras atividades. Outras 196 mulheres, com idade média de 54,9 \pm 5,9 anos, participaram da validação da forma final do questionário proposto. Quase metade delas $(46,9 \%)$ dedicava-se a atividades no lar, $10,2 \%$ eram empregadas domésticas, $7,7 \%$ eram professoras e $35,2 \%$ exerciam outras atividades. As mulheres que eram casadas ou tinham parceiro fixo resultavam em $81,1 \%$ e $6,1 \%$ eram solteiras, $12,7 \%$ viúvas ou separadas sem parceiro sexual fixo. As brancas somavam 47,9\%, negras $16,3 \%$, amarelas 5,6\% e indígenas $1 \%, 29,1 \%$ eram miscigenadas.

\section{Tamanho de amostra}

O tamanho mínimo da amostra para testar a formatação final do questionário proposto foi de quatro mulheres por item/questão, proporção de 4:1 considerada adequada por análise fatorial ou coeficiente de correlação acima de $0,2^{21}$. Assumindo t para intervalo de confiança de $95 \%$ (IC95\%<0,05), margem de erro aceitável de 4\%, variância para escala com seis pontos e quatro desvios padrão, o tamanho da amostra foi reexaminada pela fórmula de Cochran, $\mathrm{n}=(\mathrm{t})^{2}(\mathrm{~s})^{2} /(\mathrm{d})^{2}$, onde $\mathrm{t}=0,05, \mathrm{~s}=$ número de opções por item e $\mathrm{d}=$ número de itens vezes a margem de erro aceitável ${ }^{22}$.

\section{Definições e pontuação dos itens}

A definição de disfunção sexual incluiu nove domínios ou dimensões, sendo cada um definido pelo modelo de Basson. Na estrutura do questionário, cada item incluído foi adaptado segundo escala de Likert ${ }^{23}$. O questionário inicial foi desenhado com respostas dimensionais numéricas contínuas entre 0 e 5 , mais de 40 itens de mesmo peso, de modo que o escore total do questionário resulta da soma simples de todos os itens. As escalas foram transformadas em dimensões de 0 a 100, sendo que 100 indica a condição mais favorável (melhor função sexual) e 0 , a menos favorável (pior função sexual). Esta padronização linear das respostas entre 0 e 100 foi processada pelo escore dado a um determinado item menos 1 , dividido por 5 . Este resultado foi multiplicado por 100. O escore total da primeira versão do questionário foi obtido somando-se os resultados de todos os itens usando a fórmula: (x)-57/285x100, onde $\mathrm{x}$ é o item em análise, 57 o pior resultado possível e 285 o melhor resultado possivel ${ }^{24}$. Os itens negativos foram analisados ao reverso, tomando-se o maior valor, adicionando o menor valor e subtraindo-se o escore assinalado, pela fórmula: $\mathrm{QR}=5+0$-escore da $\mathrm{QR}$, valor maior + valor menor-resposta $\mathrm{QR}$ (onde $\mathrm{QR}$ corresponde à questão reversa).
Caso um item individual não tenha sido respondido, mas a respondente tenha assinalado mais de $50 \%$ dos itens daquele domínio, o valor perdido foi estimado pelo valor médio dos itens respondidos, método da meia escala, por simples imputação da média ${ }^{24}$. As questões consideradas negativas, por domínios, foram: autoimagem (Q4, Q5), qualidade de vida sexual (Q6), intimidade sexual (Q11, $\mathrm{Q} 12)$, desejo (Q21, Q22), orgasmo (Q34, Q35, Q36), dispareunia/vaginismo (Q38, Q39, Q40, Q41, Q43) e influência na menopausa (Q56).

\section{Desenvolvimento, seleção e redução de itens}

Questionários previamente validados para avaliar a função sexual em mulheres pré e pós-menopausa serviram de fonte para muitas questões que compõem este instrumento em validação ${ }^{11,13,25-33}$. Esta fase teve como objeto a classificação e análise de cada item para linguagem acessível, concordância, sintaxe, clareza e compreensão, eliminar possíveis interpretações divergentes ou ambiguidades, obter respostas corretas no formato de múltipla escolha, verificar e simplificar a aplicação das questões e examinar os escores obtidos. Os itens gerados foram agrupados por domínio, mas a sequência dos itens em cada domínio foi randomizada, por sorteio, sem reposição. Para crítica e eventuais correções na linguagem e formatação dos itens, a proposta inicial com 5 itens no domínio autoimagem, 5 na qualidade de vida sexual, 7 no domínio intimidade sexual, 9 para avaliar desejo, 7 para excitação, 4 para orgasmo, 6 para dispareunia/vaginismo, 11 em satisfação sexual e 3 na influência da menopausa, foi submetida à avaliação de sete especialistas em sexologia, sendo cinco ginecologistas, um psicólogo e um epidemiologista. Para análise inicial e possíveis correções, este questionário foi ainda aplicado a um grupo de 55 mulheres pós-menopausa. Os itens com taxas de respostas acima de $20 \%$ no extremo superior (efeito teto) ou inferior (efeito piso) foram excluídos por pouca aceitabilidade ${ }^{26}$. Itens com médias extremas e variância de zero ou quase zero também foram, por sim, excluídos por baixo poder de discriminação. Os itens mantidos nesta etapa foram, por fim, avaliados para reprodutibilidade pelo coeficiente de correlação de Pearson $(\mathrm{r})$, e os itens com coeficiente de correlação $\mathrm{r}<0,300$ foram excluídos por falta de homogeneidade ${ }^{34}$.

\section{Avaliação da confiabilidade e validação}

do questionário

A consistência interna, em cada domínio e escala total, da primeira versão com os 57 itens foi verificada pelo coeficiente $\alpha$ de Cronbach. A reprodutibilidade, ou estabilidade temporal, do questionário foi testada pela sua aplicação em dois momentos diferentes com intervalo de três semanas (teste-reteste). A correlação entre os escores obtidos no teste-reteste foi examinada pelos coeficientes 
de correlação de Pearson e coeficiente de concordância de $\operatorname{Lin}^{35}$. Após exclusão dos itens inadequados, uma segunda versão do questionário proposto com 43 itens foi aplicada a outras 196 mulheres na pós-menopausa e testada para reprodutibilidade e validades de face, conteúdo e construto. A validação teórica da face do questionário foi determinada pelo julgamento de sete especialistas em disfunção sexual, com o objetivo de verificar se de fato o instrumento é adequado para avaliar a função sexual. O exame do conteúdo, extensão pelos quais os conceitos físicos e psíquicos estão representados e examinados nos itens propostos, foi também verificado pelos mesmos especialistas ao responderem à questão: "O conteúdo medido por esta questão é essencial/útil/não essencial para avaliar a função sexual?" Exigiu-se que mais de $60 \%$ dos especialistas respondessem afirmativamente para que o item fosse considerado válido e mantido ${ }^{36}$.

\section{Análise estatística}

Os dados foram descritos como proporções, media, desvio padrão e variância. Associações foram feitas pelos coeficientes de correlação de Pearson, intraclasse e concordância de $\operatorname{Lin}^{35,36}$. A avaliação estatística das propriedades psicométricas e consistência interna foram examinadas pelo coeficiente $\alpha$ de $\mathrm{Cronbach}^{37}$. Os coeficientes de correlação/ concordância foram comparados pelo teste $t$ de Student. Valores de $\mathrm{p}<0,05$ foram considerados significantes.

\section{Resultados}

\section{Desenvolvimento, seleção, redução}

de itens do questionário

No total, 57 itens foram gerados. O painel clínico multidiciplinar composto pelos sete especialistas que analisaram a proposta inicial fez sugestões para adaptações

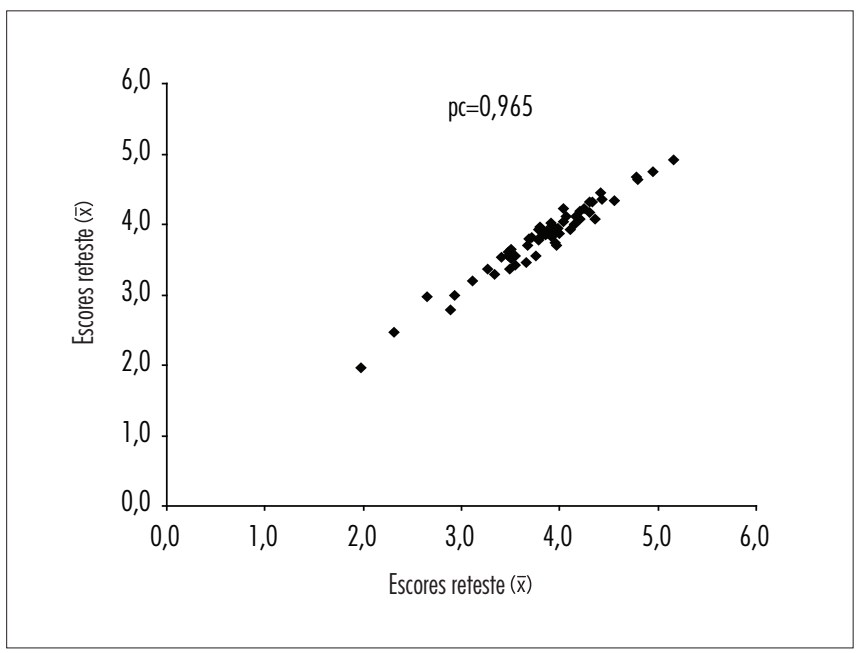

Figura 1 - Concordância dos escores (Lin, pc) obtidos no teste-reteste da primeira versão do questionário. nas questões Q1, 3, 5, 6, 12, 21, 23, 28, 30, 31, 40, 44, $46,48,52$ e 53 . Todas foram adaptadas para maior relevância clínica, segundo as sugestões recebidas. Na seleção de itens, possivelmente desconexos, com pequeno poder discriminatório, foi demonstrado que nenhuma das 57 questões da versão inicial apresentou taxa de resposta acima de $80 \%$ no extremo superior (efeito teto) ou de $20 \%$ no limite inferior (efeito piso) (dados não mostrados). Assim, nenhuma questão foi eliminada por esse critério. Do mesmo modo, nenhuma questão mostrou baixa aceitabilidade, passível de ser eliminada, indicando que a maioria das respondentes entendeu as questões e as opções das respostas. Não foi eliminado nenhum item por apresentar médias extremas e variância próxima ou igual a zero. As questões 29, 41, 46, 47, 48, 51 e 52 tiveram aprovação de apenas 25 a $60 \%$ dos especialistas e, por este motivo, foram retiradas da versão final do questionário. A questão 29 foi também eliminada por apresentar baixo coeficiente de correlação com a escala total. A correlação de escores de cada item com o escore total da escala determinou a eliminação de oito questões (Q21, Q25, Q29, Q38, Q40, Q41, Q43 e Q54) por apresentarem coeficientes de correlação $<0,300$, critério de corte para retenção e manutenção do item. Todas as outras questões mostraram correlação item-total corrigida entre 0,322 e 0,807 (dados não mostrados).

\section{Avaliação da confiabilidade e validação do questionário}

O exame de reprodutibilidade/estabilidade (teste-reteste) da primeira versão do questionário proposto demonstrou forte concordância entre as respostas iniciais e aquelas dadas após três semanas ( $\mathrm{pc}=0,965$ e $\mathrm{r}=0,882$; Figura 1 ). Nenhum item apresentou coeficiente $r<0,400$, e a maioria deles $(56,1 \%)$ alcançou $r \geq 0,700$. A comparação estatística desta correlação mostrou significância $(\mathrm{p}<0,001)$ em todos os 57 itens. Após a eliminação de 14 itens, considerados irrelevantes por terem baixo poder discriminatório ou não apresentar boa corelação com o exame total dos itens, esta versão reduzida foi aplicada a outras 196 mulheres. Esta versão final não apresentou efeitos teto ou piso em nenhum de seus itens (dados não mostrados). O escore total médio e

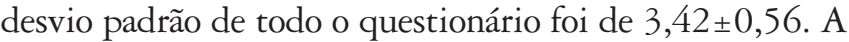
consistência interna desta formatação final mostrou coeficiente $\alpha$ de Cronbach de 0,951 (Tabela 1), demonstrando excelente confiabilidade e consistência entre os nove domínios. No entanto, os domínios autoimagem e influência da menopausa mostraram $\alpha$ de Cronbach entre 0,599 e 0,643. O domínio dispareunia/vaginismo com $\alpha$ de Cronbach de 0,371 não mostrou consistência interna satisfatória.

A validade de face, ou seja, associação lógica entre os itens e a proposta do questionário, recebeu aprovação de todos os sete especialistas que examinaram o instrumento. 
Estes não fizeram nenhuma modificação na sequência dos domínios apresentados. Em relação à validade de conteúdo, ficou claro, pela opinião dos especialistas consultados, que o objetivo do instrumento proposto de avaliar a função sexual em mulheres na pós-menopausa foi alcançado. Do mesmo modo, o questionário mostrou-se coerente em relação aos conceitos do que se pretende medir: autoimagem, qualidade de vida sexual, desejo, excitação, orgasmo, dispareunia, satisfação sexual e influência da menopausa na resposta sexual. Além disso, a importância de cada item, examinada pelas respostas de cada especialista para a alternativa essencial/útil, mas não essencial ou não necessária, mostrou taxa de aprovação $>60 \%$ em todas as questões (dados não mostrados). A validade de construto, extensão pelo qual o questionário proposto atende às hipóteses existentes a respeito do conceito (construto) que está sendo medido, neste caso função sexual, ficou demonstrada pelo coeficiente $\alpha$ de Cronbach de 0,951 (Tabela 1) e correlação de cada item com o total de itens $(0,943)$.

\section{Discussão}

Questionários produzem dados para pesquisas. A preocupação com a qualidade das informações obtidas deve ser a mesma que os investigadores têm quando utilizam dados laboratoriais. Assim, estudos epidemiológicos exigem que as informações obtidas com questionários sejam reproduzíveis, consistentes e tenham validade interna e externa. Deve-se considerar sempre a importância da formulação dos itens ou questões sobre a maneira em que são respondidas e o modo em que os dados são colhidos. Existem variações devido ao modelo de administração do questionário e à formatação individual dos itens. É extensa a literatura com diretrizes para que os epidemiologistas otimizem e desenvolvam questionários robustos.

Inconsistências têm sido observadas quando são usados instrumentos não validados. Os modelos enviados pelo correio ou preenchidos em entrevistas, mesmo validados, têm menor precisão ${ }^{20,38}$. O questionário proposto para validação neste estudo foi idealizado para examinar a função sexual da mulher especificamente na pós-menopausa e ser preenchido reservadamente pela respondente, com mínima supervisão. A interpretação dos resultados deve considerar que a participação do supervisor foi mínima e só ocorreu quando acionado pela respondente. Deve considerar, ainda, a dificuldade em avaliar a função sexual feminina, ampliada na pós-menopausa por variáveis culturais e socioeconômicas para romper a barreira do constrangimento e entrar na intimidade da mulher. As idades das pacientes que participaram da versão inicial e final do questionário não eram diferentes e os dois grupos estavam no início da sexta década de vida. Em outros estudos, com questionários incluindo pacientes nesta faixa etária, as proporções destas são de apenas $16 \%$.
Tabela 1 - Consistência interna da versão final do questionário

\begin{tabular}{lcc}
\hline Domínio & Número de iłens & Alfa de Cronbach \\
\hline Autoimagem & 5 & 0,6 \\
Qualidade sexual & 5 & 0,7 \\
Intimidade sexual & 7 & 0,8 \\
Desejo & 7 & 0,8 \\
Exciłação & 5 & 0,8 \\
Orgasmo & 4 & 0,8 \\
Dispareunia/vaginismo & 2 & 0,3 \\
Satisfação & 5 & 0,7 \\
Influência na menopausa & 3 & 0,5 \\
Tołal & 43 & 0,9 \\
\hline
\end{tabular}

As participantes do estudo final eram de varias raças, casadas ou em união estável, dedicavam-se a atividades domésticas e tinham baixa escolaridade. Este é o perfil demográfico da maioria das mulheres brasileiras nesta faixa etária ${ }^{39}$.

Esta publicação provê a validação inicial de questionário específico para avaliar a função sexual feminina após a menopausa. Considerando que os instrumentos já existentes para estudar a função/disfunção sexual foram validados em populações distintas, incluindo homens e mulheres, tanto climatéricas como não climatéricas, a proposta foi desenvolver e validar um instrumento específico para medir a função sexual feminina na pós-menopausa. Foi desenhado, então, para servir de instrumento válido na realização de estudos clínicos, com o objetivo de examinar a natureza multidimensional da função sexual feminina nesta fase da vida. E, mais importante, após a sistematização do modelo de Basson.

O processo de validação desse questionário pela análise clínica de itens teve inicío com a avaliação de sua unidade mais básica, o item ou variável, ficando atento para a clareza da linguagem, compreensão e distribuição das frequências médias, desvio padrão e variância das respostas. Este exercício atendeu às diretrizes utilizadas na validação da maior parte dos questionários atualmente disponíveis para avaliar a função sexual feminina e mostra resultados equiparáveis ${ }^{25,33}$. Os itens reuniram critérios psicométricos básicos, possíveis de serem quantificados e independentes de vieses. Na sequência, foi avaliada a relação de um item com o outro e com a escala total. A reprodutibilidade e a consistência interna do questionário também foram examinada pelos testes clássicos disponíveis para atender a estas propostas ${ }^{40}$. Por fim, validades de face, conteúdo e construto também foram verificadas e se mostraram no mesmo nível de adequação dos instrumentos recentemente validados em português ${ }^{15,16}$.

A inexistência dos efeitos teto e piso na redução do número de itens da proposta inicial demonstrou adequado poder discriminatório dos itens mantidos. A determinação para eliminar apenas sete questões pelos especialistas consultados (7/57), por não serem consideradas essenciais para avaliar a 
função sexual, indicou a excelência do conteúdo mantido na avaliação do objeto proposto ${ }^{36}$. Como os itens também não apresentaram médias extremas, foi confirmado o bom poder discriminatório das questões mantidas. A premissa de que as medidas dos itens em escala tipo Likert deveriam ter certa equivalência dentro da escala como um todo foi observada. Do mesmo modo, os desvios padrão dos escores das questões mostraram ter equivalência e distribuíram-se ao redor de 1,0 , critério recomendável para questionários julgados adequados. A assertiva de que o item deve ser linearmente relacionado ao conceito sendo medido (consistência interna do item), considerado adequado quando o item alcança $\mathrm{r} \geq 0,400$, também foi satisfatoriamente atendida ${ }^{17}$. Estes resultados são tão válidos quanto os obtidos por outros instrumentos desenhados com o mesmo objetivo em outras populações ${ }^{25,33}$.

Os itens "eu tenho falta de vontade de ter relação sexual", "eu me masturbo", "gostaria que a excitação durasse para sempre", "eu quero que minha relação melhore" e "eu não tenho dor na relação" não correlacionaram adequadamente com o questionário proposto. A falta de correlação destes itens com o escore total sugere irrelevância destes na avaliação da função sexual. Vale notar que itens semelhantes integram outros questionários ${ }^{31,32}$. As afirmações: "eu sinto minha vagina mais fechada", "eu acho minha vagina tão apertada e é impossível o pênis do meu marido (parceiro) entrar na minha vagina" não mostraram boa correlação com o total de itens do questionário, embora tenham tido boa consistência interna. Esse resultado foi inesperado, já que as questões eliminadas estão frequentemente presentes nas queixas das mulheres na pós-menopausa, à medida que envelhecem ${ }^{3,4,6}$.

A confiabilidade das respondentes, a estabilidade temporal e a homogeneidade do questionário, estimadas pelos coeficientes intraclasse e alfa de Cronbach, demonstraram a relevância deste questionário para ser utilizado como instrumento na prática clínica de avaliação da função sexual em mulheres na pós-menopausa. A excelente correlação de todos os itens com a escala total na sua forma final nivela esta escala aos diferentes instrumentos já validados para avaliar a função sexual em humanos. Entre os vários questionários validados ${ }^{25,33}$, o coeficiente $\alpha$ de Cronbach variou entre 0,410 a 0,980 , demonstração indiscutível da qualidade clínica deste instrumento em validação. $\mathrm{O}$ McCoy Female Questionnaire ${ }^{30}$, amplamente utilizado na avaliação de mulheres climatéricas com elevado grau de instrução, apresentou coeficiente $\alpha=0,770$, coeficiente este menor do que o obtido no presente estudo. As publicações sobre quais escores de consistência interna seriam satisfatórios, ou não, fornecem números variáveis. Para alguns, coeficiente $\alpha$ excessivamente alto pode indicar que o objeto da escala é muito limitado, restrito ou contenha itens redundantes ${ }^{29}$. Parece que uma consistência interna adequada para escalas com proposta de avaliação clínica e tomada de conduta tenha $\alpha \geq 0,700$. $O$ índice de função sexual feminina (FSFI), incluindo cinco domínios iguais a este questionário (desejo, excitação, orgasmo, satisfação, dor/desconforto), mostrou coeficiente $\alpha$ de 0,820 e testereteste com $\alpha$ entre 0,790 a $0,860^{32}$. Este instrumento não inclui os domínios autoimagem e qualidade de vida sexual, dimensões relevantes na definição da qualidade sexual da mulher ${ }^{7}$. Também há a limitação em avaliar o indivíduo em período limitado há quatro semanas.

Considerando que a avaliação da função sexual pode ter imprecisão nas respostas, podendo diminuir a confiabilidade e validade da informação, os autores reconhecem esta limitação na validação deste instrumento. Em adição, os autores têm consciência de que o estudo incluiu apenas mulheres heterossexuais após a menopausa, não sendo possível propor sua aplicação em mulheres homossexuais ou em outros períodos de vida. A população incluída representa, na sua maioria, camadas mais desfavoráveis da sociedade, devendo o instrumento ser reexaminado, posteriormente, em mulheres de outras classes sociais ou escolaridade. Este novo instrumento tem a vantagem de medir tanto a resposta sexual periférica (lubrificação) como a central (excitação, desejo). Tem ainda a vantagem de considerar a satisfação pessoal relacionada à sexualidade, condição necessária para o diagnóstico da maioria das categorias de disfunção sexual ${ }^{7}$.

Embora os resultados sejam preliminares, foi concluído que este questionário é confiável e pode ser aplicado mesmo em mulheres com baixa escolaridade, podendo ser usado em estudos clínicos futuros, tendo a vantagem de fazer avaliação multidimensional da função sexual. $\mathrm{O}$ instrumento apresenta excelente reprodutibilidade temporal, com intervalo de até três semanas, e excelente consistência interna entre os itens, domínios e escala total. Além disso, a escala apresentou validades de face, conteúdo e construto adequados ao seu objetivo. Os autores projetam novos estudos para examinar o poder discriminatório dos itens entre mulheres com função sexual normal e anormal, estabelecer escores capazes de discriminar tais populações e examinar a aplicabilidade do instrumento a outras populações de mulheres pósmenopausa com níveis socioeconômicos e escolaridades diferentes, para efetivamente determinar sua validade externa. Outro passo importante será sua comparação com questionários desenvolvidos após a introdução do novo sistema de classificação da disfunção sexual.

\section{Agradecimentos}

Ao Paulo Canella, Gerson Pereira Lopes, Zuleide Aparecida Félix Cabral, Marcos Antônio Bertin, Maria do Amparo R. Caridade, Jaqueline Brendler e Osvaldo M. Rodrigues Jr., pela análise crítica e colaboração na redação e seleção dos itens que compõem este questionário. 
1. Kaplan HS. Sex intimacy, and the aging process. J Am Acad Psychoanal. 1990; 18(2): 185-205.

2. DeLamater J, Hyde JS, Fong MC. Sexual satisfaction in the seventh decade of life. J Sex Marital Ther. 2008;34(5):439-54.

3. Dennerstein L, Dudley EC, Hopper JL, Burger H. Sexuality, hormones and the menopausal transition. Maturitas. 1997;26(2):83-93.

4. da Silva Lara LA, Useche B, Rosa e Silva JC, Ferriani RA, Reis RM, de Sá MF, et al. Sexuality during the climacteric period. Maturitas. 2009;62(2): 127-33.

5. Gracia CR, Sammel MD, Freeman EW, Liu L, Hollander L, Nelson DB. Predictors of decreased libido in women during the late reproductive years. Menopause. 2004; 11 (2): 144-50.

6. Goberna J, Francés L, Paulí A, Barluenga A, Gascón E. Sexual experiences during the climacteric years: what do women think about it? Maturitas. 2009;62(1):47-52.

7. Basson R, Berman J, Burnett A, Derogatis L, Ferguson D, Fourcroy J, et al. Report of the international consensus development conference on female sexual dysfunction: definitions and classifications. J Urol. 2000; 163(3):888-93.

8. Amato P. Categories of female sexual dysfunction. Obstet Gynecol Clin North Am. 2006;33(4):527-34.

9. Laumann EO, Paik A, Rosen RC. Sexual dysfunction in the United States: prevalence and predictors. JAMA. 1999;281(6):537-44.

10. Dennerstein L, Randolph J, Taffe J, Dudley E, Burger H. Hormones, mood, sexuality, and menopausal transition. Fertil Steril. 2007;77 Suppl 4:S42-8.

11. Abdo CHN. Elaboração e validação do quociente sexual - versão feminina: uma escala para avaliar a função sexual da mulher. RBM Rev Bras Med. 2006;63(9):477-82

12. Bradan AV, Araújo ALL, Nagae DKI, Takahashi LR, Formícola NR, Miyamoto WR, et al. Aspectos da sexualidade na menopausa. Arq Méd Hosp Fac Ciênc Méd Santa Casa São Paulo. 2007;52(2):39-43.

13. Rust J, Golombok S. The GRISS: a psychometric instrument for the assessment of sexual dysfunction. Arch Sex Behav. 1986; 15(2): 157-65.

14. Daker-White G. Reliable and valid self-report outcome measures in sexual (dys) function: a systematic review. Arch Sex Behav. 2002;31(2): 197-209.

15. Thiel RRC, Dambros M, Palma PCR, Thiel M, Ricceto CLZ, Ramos MF. Tradução para português, adaptação cultural e validação do Female Sexual Function Index. Rev Bras Ginecol Obstet. 2008;30(10):504-10

16. Pacagnella RC, Vieira EM, Rodrigues Júnior OM, Souza $C$. Adaptação transcultural do Female Sexual Function Index. Cad Saúde Pública. 2008;24(2):416-26.

17. Ware JE Jr, Gandek B. Methods for testing data quality, scaling assumptions, and reliability: the IQOLA Project Approach. International Quality of Life Assessment. J Clin Epidemiol. 1998;51(11):945-52

18. Lawshe $\mathrm{CH}$. A quantitative approach to content validity. Pers Psychol. 1975;28(4):563-75.

19. Prieto L, Alonso J, Lamarca R. Classical Test Theory versus Rasch analysis for quality of life questionnaire reduction. Health Qual Life Outcomes. 2003;1:27.

20. Rosen T, Olsen J. Invited commentary: the art of making questionnaires better. Am J Epidemiol. 2006;164(12): 1 145-9.
21. Bartlett JE, Kotrlik JW, Higgins CC. Organization research: determining appropriate sample in survey research. Inf Technol Learn Perform J. 2001;19(1):43-50.

22. Cochran WG. Sampling techniques. New York: John Wiley \& Sons; 1977

23. Likert R. A technique for the measurement of attitudes. Arch Psychol. 1932;22(140):55.

24. Badia X, Webb SM, Prieto L, Lara N. Acromegaly Quality of Life Questionnaire (AcroQol). Health Qual Life Outcomes. 2004;2:13.

25. Taylor JF, Rosen RC, Leiblum SR. Self-report assessment of female sexual function: psychometric evaluation on the Brief Index of Sexual Functioning for Women. Arch Sex Behav. 1994;23(6):627-43.

26. Clayton AH, McGarvey EL, Clavet GJ. The Changes in Sexual Functioning Questionnaire (CSFQ): development, realibility, and validity. Psychopharmacol Bull. 1997;33(4):731-45.

27. Mazer NA, Leiblum SR, Rosen RC. The brief index of sexual functioning for women (BISF-W): a new scoring algorithm and comparison of normative and surgically menopausal populations. Menopause. 2000;7(5):350-63.

28. Chevret M, Jaudinot E, Sullivan K, Marrel A, De Gendre AS. Quality of sexual life and satisfaction in female partners of men with ED: psychometric validation of the Index of Sexual Life (ISL) questionnaire. J Sex Marital Ther. 2004;30(3):141-55.

29. Derogatis LR. The Derogatis Interview for Sexual Functioning (DISF/DISFSR): an introductory report. J Sex Marit Ther. 1997;23(4):291-304.

30. McCoy NL. The McCoy Female Sexuality Questionnaire. Qual Life Res. 2000;9 Suppl 6:739-45.

31. Symonds T, Boolell M, Quirk F. Development of a questionnaire on sexual quality of life in women. J Sex Marit Ther. 2005;31 (5): 385-97.

32. Rosen R, Brown C, Heiman J, Leiblum S, Meston C, Shabsigh R, et al. The Female Sexual Function Index (FSFI): a multidimensional self-report instrument for the assessment of female sexual function. J Sex Marit Ther. 2000;26(2):191-208.

33. Derogatis LR, Rosen R, Leiblum S, Burnett A, Heiman J. The Female Sexual Distress Scale (FSDS): initial validation of a standardized scale for assessment of sexually related personal distress in women. J Sex Marital Ther. 2002;28(4):317-30.

34. Pesudovs K, Burr JM, Harley C, Elliott DB. The development, assessment, and selection of questionnaires. Optom Vis Sci. 2007;84(8):663-74

35. Lin LI. A concordance correlation coefficient to evaluate reproducibility. Biometrics. 1989;45(1):255-68.

36. Terwee $C B$, Bot $S D$, de Boer MR, van der Windt DA, Knol DL, Dekker J, et al. Quality criteria were proposed for measurement properties of health status questionnaires. J Clin Epidemiol. 2007;60(1):34-42.

37. Bland JM, Altman DG. Statistics notes: Cronbach's alpha. BM. 1997;314(7080):572.

38. Bowling A. Mode of questionnaire administration can have serious effects on data quality. J Public Health (Oxf). 2005;27(3):281-91.

39. de Medeiros SF, de Medeiros MM, de Oliveira VN. Climacteric complaints among very low-income women from a tropical region of Brazil. Sao Paulo Med J. 2006; 124(4):2 14-8.

40. Jensen MP. Questionnaire validation: a brief guide for readers of the research literature. Clin J Pain. 2003;19(6):345-52. 